\title{
Pain in a Man With Opioid Use Disorder Who Is Taking Medication-Assisted Therapy
}

Jessica Rich, DO' - W. Clay Jackson, MD, DipTh²

A 62-year-old White man presented to our emergency department with symptoms of right-sided facial numbness and dysarthria. He also had been having abdominal pain for the previous 5 days, which was associated with nonbloody diarrhea. His medical history was significant for extensive cardiac disease.

The patient was admitted to the hospital for observation for a transient ischemic attack and abdominal pain/ diarrhea. In the hospital, he was found to have a concomitant acute kidney injury (with a history of stage 3 chronic kidney disease) and new pulmonary edema. His neurological workup (including examination and radiographic imaging) was noncontributory. The patient was started on heparin and intravenous (IV) furosemide, his home lisinopril dose was discontinued, and all other home medications were continued.

The patient had been prescribed opioids for chronic neck pain in the distant past and had later developed opioid use disorder, for which he received medication-assisted therapy (MAT) with buprenorphine/naloxone. The patient-reported home dose of buprenorphine/naloxone was half of an 8 to $2 \mathrm{mg}$ sublingual film, which was continued upon admission to the hospital. In addition, he was started on acetaminophen as needed for breakthrough pain and acetaminophen-butalbital-caffeine for headaches.

Upon presentation, the patient had required $4 \mathrm{~L}$ of oxygen by nasal cannula (previously, he had not required home oxygen) and had dyspnea upon exertion. An echocardiography scan revealed new, severe pulmonary hypertension. A computed tomography scan conducted on hospital day 2 revealed pneumonia and pneumonitis. Because of a recent history of the ceiling falling in his house, the patient was started on IV steroids for hypersensitivity pneumonitis and antibiotics.

The patient's hospital course continued unremarkably, aside from slowly improving shortness of breath and reports of new back pain. In addition to his home dose of buprenorphine/naloxone, he was started on IV morphine, 2 mg, every 4 hours as needed on hospital day 2

\section{AFFILIATIONS:}

${ }^{1}$ Hematology \& Oncology Division, University of Tennessee College of Medicine, Memphis, Tennessee ${ }^{2}$ Director, Palliative Care Division, University of Tennessee College of Medicine, Memphis, Tennessee

\section{CITATION:}

Rich J, Jackson WC. Managing patients with pain who are taking medication for opioid use disorder. Consultant. Published online June 3, 2021. doi:10.25270/con.2021.06.00002

Received April 14, 2021. Accepted April 28, 2021.

\section{DISCLOSURES:}

The authors report no relevant financial relationships.

\section{CORRESPONDENCE:}

W. Clay Jackson, MD, DipTh, University of Tennessee College of Medicine, 920 Madison Avenue, Memphis, TN 38163 (mydocjackson@live.com)

for back pain. The morphine dose was increased to $4 \mathrm{mg}$ on hospital day 5 because of persistent back pain. His overall medical condition Improved, including resolution of the neurologic symptoms, dyspnea, and renal function. On hospital day 7 , the morphine was discontinued, and the patient began to refuse his daily buprenorphine/naloxone and request IV opioids for his back pain. Medical documentation recorded by the hospitalist showed that the patient's chronic pain was well-controlled throughout this time. The patient was subsequently started on IV hydromorphone, $0.5 \mathrm{mg}$, every 4 hours as needed on hospital day 9 .

A palliative care specialist was consulted on hospital day 10 to assist with pain management. During the palliative care assessment, the patient reported that he started having midthoracic back pain radiating distally and to his left lateral rib area, characterized as sharp and unrelenting. He related that when he had acute pain at home, he would take acetaminophen for relief. A physical examination did not reproduce the pain in the area described.

Verbal concerns were brought to the palliative care team by both nursing staff and the pharmacist regarding a concern for drug-seeking behaviors from the patient. The patient stated that he had been instructed to discontinue his buprenorphine/naloxone if he was taking any other opioid medication. The patient's addiction specialist had not been contacted by the primary team for confirmation of his medication dose or instructions for managing new, acute pain.

The patient was started on oxycodone, $15 \mathrm{mg}$, every 4 hours as needed and 
continued on IV hydromorphone, $0.5 \mathrm{mg}$, every 4 hours as needed. This regimen was initiated with instructions to try oral pain relievers prior to IV medications, as the IV would be discontinued the next day if tolerated. Prior to changing his opioid dose, the Tennessee state prescription monitoring program was accessed and confirmed the patient's outpatient buprenorphine/naloxone prescription.

Overnight, the patient continued to request IV hydromorphone after a trial of oral oxycodone, $15 \mathrm{mg}$. The next day, his oxycodone was increased to $20 \mathrm{mg}$ every 4 hours as needed, and the IV hydromorphone was discontinued by the end of the day. The patient verbalized improvement in his back pain and was discharged the following day (hospital day 15) with a 3-day supply of oxycodone, $10 \mathrm{mg}$, (1-2 tablets every 4 hours as needed) and was instructed to follow up with his addiction specialist in 3 days.

\section{Discussion}

Acute pain management remains a challenge among patients with opioid use disorder, and it has been recognized that these patients are commonly undertreated when presenting with pain.

The number of patients seeking MAT for opioid dependence is growing, and many clinicians struggle to provide adequate care for these patients who also have acute pain in the hospital setting. Multiple issues contribute to poor patient and primary physician satisfaction in appropriately treating these patients, including "opiophobia," or the fear of prescribing opioids, the intractable subjectivity of pain, and lack of adequate education in pain management, particularly for patients with a substance use disorder.

One contributing factor to the confusion is the failure to distinguish between drug-seeking behaviors resulting from uncontrolled pain and those resulting from a desire for opioid-induced euphoria. In addition, there remains considerable misunderstanding regarding the differences between dependence, tolerance, and addiction. Addiction is a "loss of control over use, craving and unwarranted preoccupation with non-therapeutic use due to craving, and continued use of the drug despite harm resulting from use,"2 while physical dependence is "an expected, neuroadaptive consequence of chronic exposure to an agonist class of drug."3 Tolerance is defined as either "a need for markedly increased amounts of a substance to achieve intoxication or a desired effect" or as "markedly diminished effect with continued use of the same amount of substance." ${ }^{13}$ Unfortunately, the poor understanding of each term leads to them being used interchangeably, contributing to the stigma of opioid use.

Patients taking MAT for a history of opioid use disorder are admitted to hospitals for various medical conditions unrelated to their pain, just as are patients not taking MAT. However, the stigma of opiophobia and lack of widespread education in the medical community about the algorithm of treatment of acute pain in patients taking MAT have created confusion and anxiety among patients and clinicians alike. Our case report highlights the basic, recurring inpatient dilemmas, elucidating the need for ongoing physician education and awareness to provide the best quality of treatment and care.

In their classic article from 2006, Alford and colleagues ${ }^{4}$ provided insight into 4 main misconceptions that inhibit quality care of acute pain in patients taking MAT:

1. Maintenance opioid agonists provide adequate analgesia for new, acute pain (they do not).

2. The use of opioids for analgesia may result in an addiction relapse (the risk of relapse is higher with the stress of unrelieved pain than it is with acute use of opioids for pain).

3. The additive effects of opioid analgesics and MAT may cause fatal respiratory and central nervous system depression (patients prescribed MAT typically develop tolerance to respiratory depression effects quickly).
4. The patient's report of pain may be a manipulation to obtain opioid medications (drug-seeking) because of opioid addiction (pain is pain and is often accentuated in the opioid use disorder population because of genetic, psychological, and pharmacologic effects).

Unfortunately, these myths continue to be prominent among health care providers.

In our patient's case, we first recognized a breakdown of interdisciplinary collaboration and communication. With a paucity of inpatient addiction specialists and pain management teams, palliative care specialists are often called in to fill in the gaps. It is important that clinicians who are trained in opioid-dose adjustments maintain baseline avoidance of withdrawal (one of the primary goals of MAT), as well as provide adequate analgesia for additional acute pain. We observed that, when our patient was given nonpharmacologic interventions, nonopioid pharmacologic interventions, and reassurance that access to opioids would be maintained if needed, he became less anxious and reported relief with oral opioid formulations. He decreased his reliance on parenteral formulations, facilitating a timely discharge.

As the number of patients taking MAT increases, with wider acceptance of therapy and more formulations available, it is paramount to establish continuity of care between the established outpatient prescriber and the inpatient team. The lack of information clarification between the patient's inpatient care team and the outpatient addiction specialist left him with the impression that continuing MAT would interfere with his analgesia, which complicated his care and delayed his discharge. Viewing the treatment of pain as that of a chronic disease with exacerbations, akin to heart failure or obstructive lung diseases, may provide a foundation of nonbias and foster an environment where hospitalists feel comfortable 
managing the basic treatment of acute pain among patients taking MAT.

In contrast to the modifiable communication and education components of treating patients taking MAT, the intractable subjectivity of patients' pain as they interface with a modern medical model, which prizes objective findings, will continue to present challenges. Studies describing multiple etiologies of pain and variability by person caused by hyperalgesia and differences in pain tolerance highlight the fact that the subjectivity of pain is part and parcel of the individual patient experience. ${ }^{5,6}$ Contrary to the belief that opioid analgesia may result in addiction relapse, studies have shown that relapse is more likely caused by poorly controlled pain or fear of withdrawal effects. ${ }^{7,8}$ Two studies by Yassen and colleagues have demonstrated that there is a ceiling effect for respiratory depression but not for analgesia by buprenorphine, alleviating fear of fatal respiratory distress with additional opioids used for further acute pain. ${ }^{9,10}$ The interpretation of what may be identified by clinicians as "drug-seeking behavior" is complex. Alford and colleagues encourage clinicians to assess objective findings and to identify patient concerns of being stigmatized or having their pain undertreated. ${ }^{4}$ Recognizing pseudoaddiction-the seeking of alternative sources and increased doses of analgesia caused by the inability to obtain tolerable relief ${ }^{11}$ and therapeutic dependence-or the fear of reemergence of pain or emergence of withdrawal symptoms ${ }^{12}$ may encourage clinicians to have an open conversation with patients regarding their pain and ask more therapy-directed questions.

Continuing education regarding federal and state laws and opioid dosing, including information regarding a subjective treatment pattern and addressing common misconceptions, may help ease clinicians' anxiety and confusion when caring for patients with acute pain who receive MAT for opioid use disorder.

\section{Conclusion}

We describe several common gaps in care that occur when patients taking MAT are admitted for inpatient treatment. Despite the current literature providing algorithms for an approach to treatment of acute pain in patients taking MAT and an increase in prescriber education regarding opioid use, many hospitalists and consultants continue to express discomfort with treating pain in this setting. As clinicians continue to prescribe MAT, it behooves us to recognize the misconceptions contributing to opiophobia that may reduce the quality of care given to these patients.

\section{References}

1. Morgan JP. American opiophobia: customary underutilization of opioid analgesics. Adv Alcohol Subst Abuse. 1985;5(1-2):163173. https://doi.org/10.1300/j251v05n01_11

2. Scimeca MM, Savage SR, Portenoy $R$, Lowinson J. Treatment of pain in methadone-maintained patients. Mt Sinai J Med. 2000;67(5-6):412-422. https://wmich.edu/ sites/default/files/attachments/u372/2015/ Treatment\%20of\%20Pain.pdf

3. Heit HA. Addiction, physical dependence, and tolerance: precise definitions to help clinicians evaluate and treat chronic pain patients. J Pain Palliat Care Pharmacother. 2003;17(1):15-29. https://doi.org/10.1080/ j354v17n01_03

4. Alford DP, Compton P, Samet JH. Acute pain management for patients receiving maintenance methadone or buprenorphine therapy. Ann Intern Med. 2006;144(2):127134. https://doi.org/10.7326/0003-4819-1442-200601170-00010

5. Doverty M, Somogyi AA, White JM, et al. Methadone maintenance patients are cross-tolerant to the antinociceptive effects of morphine. Pain. 2001;93(2):155163. https://doi.org/10.1016/s03043959(01)00306-2

6. Compton $\mathrm{P}$, Canamar $\mathrm{CP}$, Hillhouse $\mathrm{M}$, Ling $W$. Hyperalgesia in heroin dependent patients and the effects of opioid substitution therapy. J Pain. 2012;13(4):401-409. https:// doi.org/10.1016/j.jpain.2012.01.001

7. Dennis BB, Bawor M, Paul J, et al. Pain and opioid addiction: a systematic review and evaluation of pain measurement in patients with opioid dependence on methadone maintenance treatment. Curr Drug Abuse Rev. 2016;9(1):49-60. https://doi.org/10.2174 /187447370901160321102837

8. Manfredi PL, Gonzales GR, Cheville AL, Kornick C, Payne R. Methadone analgesia in cancer pain patients on chronic methadone maintenance therapy. J Pain Symptom Manage. 2001;21(2):169-174. https://doi. org/10.1016/s0885-3924(00)00252-9

9. Yassen A, Olofsen E, Romberg R, Sarton E, Danhof M, Dahan A. Mechanism-based pharmacokinetic-pharmacodynamic modeling of the antinociceptive effect of buprenorphine in healthy volunteers. Anesthesiology. 2006;104(6):1232-1242. https:// doi.org/10.1097/00000542-20060600000019

10. Yassen A, Olofsen E, Romberg R, et al. Mechanism-based PK/PD modeling of the respiratory depressant effect of buprenorphine and fentanyl in healthy volunteers. Clin Pharmacol Ther. 2007;81(1):50-58. https://doi.org/10.1038/sj.clpt.6100025

11. Weissman DE, Haddox DJ. Opioid pseudoaddiction--an iatrogenic syndrome. Pain. 1989;36(3):363-366. https://doi. org/10.1016/0304-3959(89)90097-3

12. Portenoy RK, Foley KM. Chronic use of opioid analgesics in non-malignant pain: report of 38 cases. Pain. 1986;25(2):171186. https://doi.org/10.1016/03043959(86)90091-6 